\title{
A randomized controlled clinical trial of vildagliptin plus metformin combination therapy in patients with type II diabetes mellitus
}

\author{
YONG SU, YA-LI SU, LI-FANG LV, LI-MIN WANG, QUAN-ZHONG LI and ZHI-GANG ZHAO \\ Department of Endocrinology, Henan Provincial People's Hospital, Zhengzhou University, \\ Zhengzhou, Henan 450003, P.R. China
}

Received August 22, 2013; Accepted January 15, 2014

DOI: 10.3892/etm.2014.1545

\begin{abstract}
The aim of the present study was to assess the efficacy and safety of vildagliptin plus metformin combination therapy in patients with type II diabetes mellitus. Type II diabetic patients with poor glycemic control following at least three months of metformin treatment were selected and randomized into two groups. Vildagliptin or placebo was administered with metformin. Body weight, fasting blood glucose (FBG), postprandial glucose (PPG), glycated hemoglobin (HbAlc), blood lipid and hepatorenal function levels were analyzed in the patients prior to and 24-weeks after the trial. FBG, PPG and $\mathrm{HbA} 1 \mathrm{c}$ levels of the patients in the vildagliptin group significantly decreased following the trial, whereas no statistically significant differences were observed in the various indicators of the placebo group prior to and following the trial. The FBG, PPG and HbA1c levels in the vildagliptin group were significantly lower compared with the placebo group 24-weeks after the trial. Comparisons of body weight, blood lipid and hepatorenal function between the groups prior to and following the trial exhibited no statistically significant differences. Therefore, vildagliptin plus metformin combination therapy effectively reduced FBG, PPG and HbA1c levels in patients with no risk of weight gain or hepatorenal dysfunction.
\end{abstract}

\section{Introduction}

The incidence of type II diabetes mellitus is gradually increasing. According to the International Diabetes Federation (1), the number of diabetic patients worldwide is likely to reach 552 million in 2030, in which $90 \%$ of these patients have type II diabetes.

Correspondence to: Dr Ya-Li Su, Department of Endocrinology, Henan Provincial People's Hospital, Zhengzhou University, Zhengzhou, Henan 450003, P.R. China

E-mail: yalisucn@163.com

Key words: type II diabetes mellitus, vildagliptin, metformin, body weight, blood lipid, hepatorenal function
Vildagliptin is a highly selective, reversible and competitive dipeptidyl peptidase-4 (DPP-4) inhibitor. The efficacy of vildagliptin plus metformin combination therapy has been demonstrated to lower the risk of weight gain and hypoglycemia and significantly decrease fasting blood glucose (FBG), postprandial glucose (PPG) and glycated hemoglobin (HbAlc) levels. No evidence has shown that vildagliptin use carries the risk of hepatorenal dysfunction (2-7). In 2009, the American Society for Clinical Endocrinology released the diabetes control guidelines $(8,9)$, which indicate DPP-4 inhibitors as first-line drugs for type II diabetes. In addition, according to the guidelines, if metformin or thiazolidinediones do not produce favorable results, a DPP-4 inhibitor may be used in combination to support the therapy.

However, no associated studies exist regarding this combination therapy in type II diabetes patients in China. Thus, in the present study, the effects of vildagliptin plus metformin combination therapy in decreasing the blood glucose level were analyzed. In addition, the effects of this therapy on body weight and hepatorenal function of patients were analyzed.

\section{Materials and methods}

Subjects. A total of 600 patients (males, 324; females, 276) were involved in the trial. The study was conducted in accordance with the Declaration of Helsinki and with approval from the Ethics Committee of Henan Provincial People's Hospital (Zhengzhou, China). Written informed consent was provided by all participants.

Inclusion criteria. Patients diagnosed with type II diabetes with a 75-g oral glucose tolerance test that were aged between 18 and 70 years-old were included in the study. In addition, the included patients had been treated with metformin and A-glycosidase inhibitors for longer than three months, had HbAlc levels $>6.5 \%$, FBG levels $>7.0 \mathrm{mmol} / \mathrm{l}$ and normal hepatorenal function. All subjects volunteered to participate in this study and provided informed consent.

Exclusion criteria. Patients excluded from the study had type I or other specific forms of diabetes, were younger than 18 or older than 70 years-old or had used hypoglycemic agents other 
Table I. Clinical characteristics of the subjects.

\begin{tabular}{|c|c|c|c|c|}
\hline Characteristics & Vildagliptin group (mean \pm SD) & Placebo group (mean $\pm \mathrm{SD})$ & t-value & P-value \\
\hline Age, years & $47.61 \pm 14.409$ & $48.67 \pm 11.564$ & -0.215 & 0.813 \\
\hline Height, $\mathrm{cm}$ & $165.50 \pm 6.456$ & $164.61 \pm 6.456$ & 0.778 & 0.486 \\
\hline Weight, kg & $65.14 \pm 10.317$ & $63.67 \pm 9.794$ & 0.335 & 0.656 \\
\hline FBG, mmol/l & $9.22 \pm 1.691$ & $8.86 \pm 1.445$ & 0.864 & 0.389 \\
\hline MPPG, mmol/1 & $10.23 \pm 1.657$ & $10.33 \pm 1.395$ & 0.038 & 0.883 \\
\hline $\mathrm{HbA1c}, \%$ & $8.76 \pm 1.718$ & $8.42 \pm 1.368$ & 0.343 & 0.671 \\
\hline ALT, U/1 & $30.44 \pm 15.846$ & $23.38 \pm 9.928$ & 1.626 & 0.113 \\
\hline AST, U/1 & $22.89 \pm 7.676$ & $20.67 \pm 5.246$ & 1.017 & 0.327 \\
\hline $\mathrm{CHOL}, \mathrm{mmol} / \mathrm{l}$ & $4.96 \pm 1.009$ & $4.53 \pm 1.096$ & 1.001 & 0.323 \\
\hline $\mathrm{TG}, \mathrm{mmol} / \mathrm{l}$ & $1.93 \pm 1.383$ & $1.85 \pm 1.373$ & 0.176 & 0.834 \\
\hline $\mathrm{HDL}, \mathrm{mmol} / \mathrm{l}$ & $1.18 \pm 0.331$ & $1.08 \pm 0.287$ & 1.420 & 0.178 \\
\hline LDL, mmol/l & $2.77 \pm 0.671$ & $2.45 \pm 0.836$ & 1.308 & 0.213 \\
\hline UREA, mmol/l & $5.58 \pm 1.652$ & $5.67 \pm 1.366$ & -0.181 & 0.749 \\
\hline CREA, $\mu \mathrm{mol} / 1$ & $57.18 \pm 13.367$ & $60.57 \pm 20.359$ & -0.590 & 0.559 \\
\hline
\end{tabular}

SD, standard deviation; FBG, fasting blood glucose; MPPG, mean postprandial glucose; HbA1c, glycated hemoglobin; ALT, alanine aminotransferase; AST, aspartate aminotransferase; CHOL, total cholesterol; TG, total triglycerides; HDL, high density lipoproteins; LDL, low density lipoproteins; UREA, urea; CREA, creatinine.

than metformin and A-glycosidase inhibitors. Patients with diabetic ketoacidosis, hyperglycemic nonketotic hyperosmolar syndrome, chronic complications that required insulin treatment or patients that required insulin treatment under stress were also excluded. Patients with evident liver or kidney disease [alanine aminotransferase (ALT) or aspartate aminotransferase (AST) levels $>3$ times the normal upper limit, total bilirubin levels $>1.5$ times the normal upper limit or creatinine (CREA) levels $>115 \mu \mathrm{mol} / 1$ ], pregnant or lactating women and patients with poor compliance were also excluded from the study.

Diet design. Participants received individual diet guidance from an endocrinologist. Total daily calorie intake from food, including $50 \%$ carbohydrate, $15 \%$ protein and $35 \%$ fat, for each subject was calculated according to $30 \mathrm{kcal} / \mathrm{kg}$ of ideal weight [ideal weight $(\mathrm{kg})=$ measured weight $(\mathrm{kg})-105]$. Each subject was assigned to three meals with calorie intake assigned as follows: $1 / 5,2 / 5$ and 2/5. Mealtimes and meal content were relatively uniform, and intake of alcohol, strong tea or coffee was prohibited during the trial.

Drug treatment. Subjects randomly received vildagliptin (100 mg/day orally in two equal doses) or placebo and received drugs according to prescription. Patients who forgot or adjusted drug doses or treatment were excluded from the trial. Patients self-monitored and recorded fingerstick blood glucose and hypoglycemic events. The body weight, FBG, PPG, HbAlc, blood lipid and hepatorenal function levels of the patients 24-weeks after the trial were analyzed. Drugs were supplied by Henan Pharmaceutical Co., Ltd. (Zhengzhou, China).

Statistical analysis. Data are presented as mean \pm SD. The data of the groups obtained before and after the trial were compared using a Student's t-test. A paired t-test was used to compare associated indicators prior to and following the trial. Data were analyzed with SPSS 13.0 software (SPSS, Inc., Chicago, IL, USA) and $\mathrm{P}<0.05$ was considered to indicate a statistically significant difference.

\section{Results}

Clinical characteristics. A total of 600 patients (males, 324; females, 276), including 300 in the vildagliptin group and 300 in the placebo group, were included in the study. The patients had an average age of $48.14 \pm 12.986$ years (18-70 years), body weight of $64.40 \pm 10.057 \mathrm{~kg}(45-80 \mathrm{~kg}), \mathrm{FBG}$ level of $9.04 \pm 1.568 \mathrm{mmol} / \mathrm{l}(7.0-12.0 \mathrm{mmol} / \mathrm{l})$, mean postprandial glucose (MPPG) level of 10.28 $\pm 1.568 \mathrm{mmol} / 1$ (7.0-13.0 $\mathrm{mmol} / \mathrm{l}$ ) and HbAlc level of $8.59 \pm 1.543 \%$ (6.5-11.6\%). In total, 14 patients quit the trial: Four violated the treatment and two were lost to follow-up in the vildagliptin group, while five violated the treatment, two were lost to follow-up and one had an adverse event in the placebo group. A total of 294 and 292 patients completed the clinical trial in the vildagliptin and placebo groups, respectively.

Table I shows that there were no statistically significant differences in age, body weight, FBG, MPPG, HbA1c, blood lipid or hepatorenal function between the two groups $(\mathrm{P}>0.05)$.

Comparison of associated indicators. The patients underwent examinations 24-weeks after the trial. In the vildagliptin group, the FBG, MPPG and HbA1c levels significantly decreased $(\mathrm{P}<0.05)$, whereas the body weight, blood lipid and hepatorenal function did not significantly change following the trial $(\mathrm{P}>0.05)$. No statistically significant differences were observed in the various indicators in the placebo group before and after the trial (Table II). 
Table II. Comparison of associated indicators before and after vildagliptin or placebo treatments.

\begin{tabular}{|c|c|c|c|c|}
\hline Indicator & Before treatment (mean \pm SD) & After treatment $($ mean $\pm \mathrm{SD})$ & t-value & P-value \\
\hline \multicolumn{5}{|c|}{ Vildagliptin group $(n=294)$} \\
\hline Weight, $\mathrm{kg}$ & $65.14 \pm 10.317$ & $65.84 \pm 10.454$ & 0.066 & 0.935 \\
\hline $\mathrm{FBG}, \mathrm{mmol} / \mathrm{l}$ & $9.22 \pm 1.691$ & $6.52 \pm 0.702$ & 6.288 & $<0.0001$ \\
\hline MPPG, mmol/1 & $10.23 \pm 1.657$ & $8.13 \pm 0.635$ & 6.040 & $<0.0001$ \\
\hline HbA1c, $\%$ & $8.76 \pm 1.718$ & $6.65 \pm 1.251$ & 4.261 & $<0.0001$ \\
\hline ALT, U/1 & $30.44 \pm 15.846$ & $33.94 \pm 11.275$ & 2.343 & 0.129 \\
\hline AST, U/1 & $22.89 \pm 7.676$ & $27.23 \pm 6.857$ & 1.245 & 0.251 \\
\hline CHOL, mmol/l & $4.96 \pm 1.009$ & $4.50 \pm 0.882$ & 1.517 & 0.138 \\
\hline $\mathrm{TG}, \mathrm{mmol} / \mathrm{l}$ & $1.93 \pm 1.383$ & $1.36 \pm 0.853$ & 1.539 & 0.133 \\
\hline HDL, mmol/1 & $1.18 \pm 0.331$ & $1.15 \pm 0.239$ & 0.385 & 0.713 \\
\hline LDL, mmol/l & $2.77 \pm 0.671$ & $2.58 \pm 0.731$ & 1.267 & 0.214 \\
\hline UREA, mmol/1 & $5.58 \pm 1.652$ & $5.91 \pm 2.089$ & -0.370 & 0.724 \\
\hline CREA, $\mu \mathrm{mol} / 1$ & $57.18 \pm 13.367$ & $57.21 \pm 14.564$ & 0.036 & 0.972 \\
\hline \multicolumn{5}{|c|}{ Placebo group $(n=292)$} \\
\hline Weight, kg & $63.67 \pm 9.794$ & $64.69 \pm 9.381$ & -0.009 & 0.993 \\
\hline $\mathrm{FBG}, \mathrm{mmol} / \mathrm{l}$ & $8.86 \pm 1.445$ & $8.60 \pm 1.274$ & 0.573 & 0.572 \\
\hline MPPG, mmol/1 & $10.33 \pm 1.395$ & $9.70 \pm 1.348$ & 1.539 & 0.136 \\
\hline HbA1c, $\%$ & $8.42 \pm 1.368$ & $8.26 \pm 1.180$ & 1.061 & 0.296 \\
\hline ALT, U/1 & $23.38 \pm 9.928$ & $26.89 \pm 8.065$ & -1.198 & 0.249 \\
\hline AST, U/1 & $20.67 \pm 5.246$ & $23.67 \pm 4.666$ & -1.867 & 0.071 \\
\hline CHOL, mmol/1 & $4.53 \pm 1.096$ & $4.77 \pm 0.950$ & -0.132 & 0.904 \\
\hline $\mathrm{TG}, \mathrm{mmol} / \mathrm{l}$ & $1.85 \pm 1.373$ & $1.99 \pm 1.327$ & -0.099 & 0.932 \\
\hline HDL, mmol/1 & $1.08 \pm 0.287$ & $1.24 \pm 0.501$ & -1.992 & 0.054 \\
\hline LDL, mmol/l & $2.45 \pm 0.836$ & $2.67 \pm 0.812$ & -0.468 & 0.657 \\
\hline UREA, mmol/1 & $5.67 \pm 1.366$ & $5.56 \pm 1.419$ & 0.922 & 0.363 \\
\hline CREA, $\mu \mathrm{mol} / 1$ & $60.57 \pm 20.359$ & $64.61 \pm 21.947$ & -0.559 & 0.580 \\
\hline
\end{tabular}

SD, standard deviation; FBG, fasting blood glucose; MPPG, mean postprandial glucose; HbA1c, glycated hemoglobin; ALT, alanine aminotransferase; AST, aspartate aminotransferase; CHOL, total cholesterol; TG, total triglycerides; HDL, high density lipoproteins; LDL, low density lipoproteins; UREA, urea; CREA, creatinine.

Table III. Comparison of indicators between vildagliptin and placebo groups 24-weeks after the trial (mean \pm SD).

\begin{tabular}{|c|c|c|c|c|}
\hline Indicator & Vildagliptin group $(n=294)$ & Placebo group $(n=292)$ & t-value & $\mathrm{P}$-value \\
\hline Weight, kg & $65.84 \pm 10.454$ & $64.69 \pm 9.381$ & 0.367 & 0.709 \\
\hline $\mathrm{FBG}, \mathrm{mmol} / \mathrm{l}$ & $6.52 \pm 0.702$ & $8.60 \pm 1.274$ & -6.125 & $<0.0001$ \\
\hline MPPG, mmol/1 & $8.13 \pm 0.635$ & $9.70 \pm 1.348$ & -4.785 & $<0.0001$ \\
\hline $\mathrm{HbA1c}, \%$ & $6.65 \pm 1.251$ & $8.26 \pm 1.180$ & -3.743 & 0.001 \\
\hline ALT, U/1 & $33.94 \pm 11.275$ & $26.89 \pm 8.065$ & 2.351 & 0.427 \\
\hline $\mathrm{AST}, \mathrm{U} / 1$ & $27.23 \pm 6.857$ & $23.67 \pm 4.666$ & 2.238 & 0.123 \\
\hline $\mathrm{CHOL}, \mathrm{mmol} / \mathrm{l}$ & $4.50 \pm 0.882$ & $4.77 \pm 0.950$ & -0.544 & 0.563 \\
\hline $\mathrm{TG}, \mathrm{mmol} / \mathrm{l}$ & $1.36 \pm 0.853$ & $1.99 \pm 1.327$ & -1.718 & 0.097 \\
\hline HDL, mmol/l & $1.15 \pm 0.239$ & $1.24 \pm 0.501$ & -0.987 & 0.342 \\
\hline $\mathrm{LDL}, \mathrm{mmol} / 1$ & $2.58 \pm 0.731$ & $2.67 \pm 0.812$ & -0.360 & 0.732 \\
\hline UREA, mmol/1 & $5.91 \pm 2.089$ & $5.56 \pm 1.419$ & 0.937 & 0.358 \\
\hline CREA, $\mu \mathrm{mol} / 1$ & $57.21 \pm 14.564$ & $64.61 \pm 21.947$ & -1.308 & 0.235 \\
\hline
\end{tabular}

SD, standard deviation; FBG, fasting blood glucose; MPPG, mean postprandial glucose; HbA1c, glycated hemoglobin; ALT, alanine aminotransferase; AST, aspartate aminotransferase; CHOL, total cholesterol; TG, total triglycerides; HDL, high density lipoproteins; LDL, low density lipoproteins; UREA, urea; CREA, creatinine. 
Comparison of efficacy. Table III shows that 24-weeks after the trial, the FBG, PPG and HbAlc levels in the vildagliptin group were significantly lower compared with the placebo group $(\mathrm{P}<0.05)$. Comparisons of body weight, blood lipid and hepatorenal function between the groups exhibited no statistically significant differences.

\section{Discussion}

Vildagliptin is a highly selective and reversible substrate-like DPP-4 inhibitor that binds to and forms a complex with DPP-4 to inhibit DPP-4 activity, increase active glucagon peptide-1 (GLP-1) concentration, promote glucose-dependent insulin secretion from pancreatic $\beta$ cells and simultaneously adjust the function of $\alpha$ cells to reduce glucagon levels during hyperglycemia (8) to achieve bidirectional regulation of blood sugar. Vildagliptin plus metformin combination therapy improves glycemic control in type II diabetic patients with favorable safety and tolerance levels $(2,5)$. Metformin may increase GLP-1 synthesis, whereas vildagliptin inhibits DPP-4 and increases activated GLP-1 levels. Therefore, the synergistic effect of vildagliptin and metformin maximizes activated GLP-1 levels and further increases insulin secretion and sensitivity (10).

The efficacy of vildagliptin plus metformin combination therapy has been reported in numerous studies in various countries $(4,5,11-18)$. However, this therapy has not been reported in China. Thus, the aim of the present clinical trial was to observe any decrease in blood glucose level caused by this combined therapy, as well as any adverse effects of vildagliptin. During the study, two cases of hypoglycemia and six incomplete cases in the vildagliptin group were observed. By contrast, in the placebo group, no hypoglycemia case was observed and eight patients quit.

The present study revealed that vildagliptin treatment markedly decreased FBG, PPG and HbAlc levels in patients when compared with patients prior to vildagliptin treatment or following placebo treatment (Tables II and III). In addition, no significant change in body weight was observed. The results indicate that the total cholesterol (CHOL), triglyceride (TG), high density lipoprotein (HDL) and low density lipoprotein (LDL) levels of patients decreased and the ALT, AST, UREA and creatine (CREA) levels increased following vildagliptin treatment.

Bidirectional regulation of vildagliptin not only reduces FBG levels, but also reduces PPG to reduce HbAlc levels effectively. Retrospective studies have demonstrated that DPP-4 inhibitor levels correlate with decreased CHOL (19). No correlation was observed between vildagliptin and increased risk of adverse liver conditions or elevated hepatase $(3,7,20,21)$. The present study demonstrated that CHOL, TG, HDL and LDL levels decreased and hepatase levels slightly increased following vildagliptin treatment, which may be attributed to the small sample size or the effect of a combination of hypoglycemic agents. However, no statistically significant differences were observed in these indicators before and after vildagliptin treatment or after placebo treatment. These results indicate that an increase in ALT due to vildagliptin intake does not negatively affect the liver function of patients. In addition, both UREA and CREA levels increased following vildagliptin treatment but were not statistically different from those prior to vildagliptin treatment. This result indicates that vildagliptin does not negatively affect the kidney function of patients.

In conclusion, vildagliptin plus metformin combination therapy effectively reduces the FBG, PPG and HbAlc levels and increases the hepatase and CREA levels of patients with type II diabetes. The combination therapy does not negatively affect hepatorenal functions, but reduces blood lipid level with a low risk of weight gain and hypoglycemia. Independent of safety or efficacy, vildagliptin may be considered as a useful hypoglycemic drug for type II diabetic patients with poor glycemic control following metformin monotreatment.

\section{References}

1. Whiting DR, Guariguata L, Weil C and Shaw J: IDF diabetes atlas: global estimates of the prevalence of diabetes for 2011 and 2030. Diabetes Res Clin Pract 94: 311-321, 2011.

2. Bosi E, Dotta F, Jia Y and Goodman M: Vildagliptin plus metformin combination therapy provides superior glycaemic control to individual monotherapy in treatment-naive patients with type 2 diabetes mellitus. Diabetes Obes Metab 11: 506-515, 2009.

3. Ligueros-Saylan M, Foley JE, Schweizer A, Couturier A and Kothny W: An assessment of adverse effects of vildagliptin versus comparators on the liver, the pancreas, the immune system, the skin and in patients with impaired renal function from a large pooled database of Phase II and III clinical trials. Diabetes Obes Metab 12: 495-509, 2010.

4. Filozof C and Gautier JF: A comparison of efficacy and safety of vildagliptin and gliclazide in combination with metformin in patients with Type 2 diabetes inadequately controlled with metformin alone: a 52-week, randomized study. Diabet Med 27: 318-326, 2010.

5. Matthews DR, Dejager S, Ahren B, et al: Vildagliptin add-on to metformin produces similar efficacy and reduced hypoglycaemic risk compared with glimepiride, with no weight gain: results from a 2-year study. Diabetes Obes Metab 12: 780-789, 2010.

6. Schweizer A, Dejager S, Foley JE, Shao Q and Kothny W: Clinical experience with vildagliptin in the management of type 2 diabetes in a patient population $\geq 75$ years: a pooled analysis from a database of clinical trials. Diabetes Obes Metab 13: 55-64, 2011.

7. Patel B and Shah J: Safety and efficacy of vildagliptin: dipeptidyl peptidase-4 inhibitor. IJPRBS 2: 35-51, 2012.

8. Ahrén B, Schweizer A, Dejager S, Villhauer EB, Dunning BE and Foley JE: Mechanisms of action of the dipeptidyl peptidase-4 inhibitor vildagliptin in humans. Diabetes Obes Metab 13: 775-783, 2011.

9. Rodbard HW, Jellinger PS, Davidson JA, et al: Statement by an American Association of Clinical Endocrinologists/American College of Endocrinology consensus panel on type 2 diabetes mellitus:an algorithm for glycemic control. Endocrine Practice 15: 540-549, 2009.

10. Mathieu C: The scientific evidence: vildagliptin and the benefits of islet enhancement. Diabetes Obes Metab 11 (Suppl 2): S9-S17, 2009.

11. Ferrannini E, Fonseca V, Zinman B, et al: Fifty-two-week efficacy and safety of vildagliptin vs. glimepiride in patients with type 2 diabetes mellitus inadequately controlled on metformin monotherapy. Diabetes Obes Metab 11: 157-166, 2009.

12. Bosi E, Camisasca RP, Collober C, Rochotte E and Garber AJ: Effects of vildagliptin on glucose control over 24 weeks in patients with type 2 diabetes inadequately controlled with metformin. Diabetes Care 30: 890-895, 2007.

13. Ahrén B, Pacini G, Tura A, Foley JE and Schweizer A: Improved meal-related insulin processing contributes to the enhancement of B-cell function by the DPP-4 inhibitor vildagliptin in patients with type 2 diabetes. Horm Metab Res 39: 826-829, 2007.

14. Bolli G, Dotta F, Rochotte E and Cohen SE: Efficacy and tolerability of vildagliptin vs. pioglitazone when added to metformin: a 24-week, randomized, double-blind study. Diabetes Obes Metab 10: 82-90, 2008. 
15. Bolli G, Dotta F, Colin L, Minic B and Goodman M: Comparison of vildagliptin and pioglitazone in patients with type 2 diabetes inadequately controlled with metformin. Diabetes Obes Metab 11: 589-595, 2009.

16. D'Alessio DA, Denney AM, Hermiller LM, et al: Treatment with the dipeptidyl peptidase-4 inhibitor vildagliptin improves fasting Islet-cell function in subjects with type 2 diabetes. J Clin Endocrinol Metab 94: 81-88, 2009.

17. Blonde L, Dagogo-Jack S, Banerji MA, et al: Comparison of vildagliptin and thiazolidinedione as add-on therapy in patients inadequately controlled with metformin: results of the GALIANT trial - a primary care, type 2 diabetes study. Diabetes Obes Metab 11: 978-986, 2009.
18. Ahrén B, Foley JE and Bosi E: Clinical evidence and mechanistic basis for vildagliptin's action when added to metformin. Diabetes Obes Metab 13: 193-203, 2011

19. Richard KR, Shelburne JS and Kirk JK: Tolerability of dipeptidyl peptidase-4 inhibitors: a review. Clin Ther 33: 1609-1629, 2011.

20. Lukashevich V, Schweizer A, Shao Q, Groop PH and Kothny W: Safety and efficacy of vildagliptin versus placebo in patients with type 2 diabetes and moderate or severe renal impairment: a prospective 24-week randomized placebo-controlled trial. Diabetes Obes Metab 13: 947-954, 2011.

21. Kalra S: Emerging role of dipeptidyl peptidase-IV (DPP-4) inhibitor vildagliptin in the management of type 2 diabetes. J Assoc Physicians India 59: 237-245, 2011. 